\title{
CLIC1 Expression in Skin Biopsies from Patients With Rheumatoid and Psoriatic Arthritis as a Potential Tool to Predict Therapy Response
}

\author{
LILIANA BORDEAN ${ }^{1,2,3}$, MONICA CHIS ${ }^{1,2}$, MARIUS RAICA ${ }^{4,5}$, OVIDIU SIMION COTOI ${ }^{6}$, \\ AMALIA RALUCA CEAUSU ${ }^{4,5}$, CLAUDIU AVRAM ${ }^{7}$ and ANCA MARIA CIMPEAN ${ }^{4,5}$ \\ ${ }^{1}$ Department ME2/Rheumatology, Rehabilitation, Physical Medicine and Balneology, Faculty of Medicine, \\ George Emil Palade University of Medicine, Pharmacy, Science, \\ and Technology of Târgu Mureş (UMPhST), Târgu Mureș, Romania; \\ ${ }^{2}$ Clinic of Rheumatology, Emergency County Hospital of Târgu Mureş, Târgu Mureș, Romania; \\ ${ }^{3}$ Doctoral School, Victor Babes University of Medicine and Pharmacy Timisoara, Timisoara, Romania; \\ ${ }^{4}$ Department of Microscopic Morphology/Histology, \\ Victor Babes University of Medicine and Pharmacy Timisoara, Timisoara, Romania; \\ ${ }^{5}$ Angiogenesis Research Center, Victor Babes University of Medicine and Pharmacy Timisoara, Timisoara, Romania; \\ ${ }^{6}$ Department M2/Physiopathology, George Emil Palade University of Medicine, \\ Pharmacy, Science, and Technology of Târgu Mureş (UMPhST), Târgu Mureș, Romania; \\ ${ }^{7}$ Physical Therapy and Special Motricity Department, West University of Timisoara, Timisoara, Romania
}

\begin{abstract}
Background/Aim: Chloride intracellular channel protein 1 (CLIC1) activates inflammasomes in rheumatoid (RA) and psoriatic (PSA) arthritis. We studied CLIC1 expression in RA and PSA patients' skin with vasculitis and its variability depending on the therapy used. Materials and Methods: CLIC1 immunoexpression was evaluated in the vascular (CLIC1-V) and stromal (CLIC1-S) compartments of the RA and PsA skin biopsies of patients treated with methotrexate (MTX), leflunomid (LFN), corticotherapy (CT), or biological therapies. Results: MTX significantly reduced CLIC1-S expression $(p=0.016)$, whereas LFN decreased CLIC1-V $(p<0.001)$. LFN therapy duration also correlated with CLIC1-V $(p<0.001)$. CT decreased CLIC1-S expression $(p=0.006)$. CLIC1-S expression persisted in skin biopsies despite of erythrocyte sedimentation rate ( $E S R, p=0.018)$ and $C$ reactive protein $(C R P, p=0.0026)$ normalisation. For
\end{abstract}

This article is freely accessible online.

Correspondence to: Monica Chiș, MD, Ph.D., Department ME2/Rheumatology, Rehabilitation, Physical Medicine and Balneology, Faculty of Medicine, George Emil Palade University of Medicine, Pharmacy, Science, and Technology of Târgu Mureş (UMPhST), Târgu Mureș, Romania. Tel: +40 725511508, e-mail: monica.copotoiu@umfst.ro

Key Words: CLIC1, skin biopsies, rheumatoid arthritis, psoriatic arthritis, therapy.
PsA, CLIC1-S expression significantly related to MTX $(p<0.022)$. Both CLIC1-S $(p<0.001)$ and CLIC1-V $(p=0.007)$ decreased by biological therapies in RA. Conclusion: CLIC1 expression is strongly influenced by the therapy used. Our data strongly support the extensive evaluation of CLIC1 in $R A$ as a potential marker of inflammation and tool to predict therapy response.

Chloride intracellular channel protein 1 (CLIC1) is a member of the CLICs family, and one of the most conserved proteins (1). It was first cloned due to its increased expression in activated macrophages (2). CLIC1, also known as NCC27, has an early and variable expression in human fetal tissues, and is found in low levels in human fetal brain but in high levels in the human fetal lung, kidney, and liver (3). During human adult life, expression variability persists among most human tissues (4). The intracellular chloride channels are membrane bound and participate in different pathological processes including inflammation associated with neurodegenerative diseases (5)atherosclerosis (6) ankylosing spondylitis (7) tumor progression, and metastasis of urinary bladder cancer (8) renal cell carcinomas (9) or hepatocellular carcinomas (10). CLIC1 has a high translocation ability between the cytoplasmic and nuclear compartments depending on the functional status and based on this translocation; CLIC1 functions are extremely versatile (11). These versatile functions of chloride channels such as CLIC1 include the regulation of cellular metabolism by acting at the 
enzymatic level (12). The role of CLIC1 in inflammation associated with different pathologies is well known. In this context CLIC1 is highly expressed in macrophages, the key cells of adaptive and innate immunity. CLIC1 expression is not restricted only to macrophages, it is also observed in connective tissue fibroblasts and myofibroblasts, abundantly found in different inflammatory lesions. The effects of CLIC1 on stromal cells are mediated by the presence of transforming growth factor beta (TGF $\beta$ ) and tumor necrosis factor alpha (TNF $\alpha$ ). In rheumatic diseases, CLIC1 is poorly studied, and the experimental models mainly include macrophages or stromal fibroblasts isolated from mice (13).

In rheumatic diseases, CLIC1 is closely correlated with the induction of inflammasomes that support the inflammation. CLIC1 is not only over-expressed in stromal cells, but also in epithelial cells such as endothelial or epidermal cells (12).

The methods of evaluating conventional and targeted therapies in rheumatic diseases are limited and the reported results are often variable. Musculoskeletal ultrasonography and synovial biopsies could not correctly evaluate the effects of the applied therapies, which is why the use of other diagnostic methods and therapeutic evaluation tools are required in rheumatoid arthritis (RA) and psoriatic arthritis (PsA) (14). Changes in the skin induced by RA or PsA are scarcely studied $(15,16)$. Data from the literature regarding the reactivity of the skin in RA and PsA before or after therapy are also extremely limited (17). Moreover, there is no specific marker that clearly characterizes skin or synovial response to therapy. CLIC1 stimulates endothelial injury, vascular inflammation, and oxidative stress, basic components in the pathophysiology of RA or PsA (18). Recent data have shown that CLIC1 inhibition attenuates the accumulation of inflammatory cytokines and reduces oxidative stress (18). Currently, for human subjects, we have no strong evidence regarding the involvement of CLIC1 in the pathophysiology of RA and PsA and its response to targeted or conventional therapy. Moreover, there are no data indicating that CLIC1 expression in skin biopsies from patients with RA and PsA supports the use of skin biopsies as a method of evaluating therapy response. Therefore, the present study aimed to evaluate CLIC1 expression in skin biopsies from RA and PsA patients administered various first/second line treatments or biological therapies.

\section{Materials and Methods}

Patients and biopsies. Thirty skin biopsies were collected from patients aged between 20 and 60 years old previously diagnosed with RA (22 skin biopsies) and PsA (8 skin biopsies) who had clinical signs of vasculitis. An informed consent was obtained from each patient before the procedure. The study was performed by respecting all ethical issues regarding the use of human tissues and the manipulation of paraffin embedded specimens and approval was obtained from the County Clinical Ethic Committee (Approval No. 2654/19.02.2019). All patients were previously treated for RA and PsA by using common treatments for these diseases associated or not with biological therapies. We selected 30 patients who received methotrexate (MTX), $20 \mathrm{mg} /$ week as first line therapy (FLIT) followed by second line therapy (SLIT) with leflunomide (LFN), 20 $\mathrm{mg} /$ day. In addition, corticotherapy was used when necessary, with $7.5 \mathrm{mg}$ /day prednisone, for at least 14 days in 10 out of 30 patients. Furthermore, 6 out of 30 patients received biological therapy with Humira $40 \mathrm{mg} /$ bimonthly and Tofacitinib $10 \mathrm{mg} /$ day. Therapies used for the treatment of RA and PsA were statistically evaluated with regard to CLIC1 immunoexpression in skin biopsy stromal and vascular compartments. Patients' data included age, diagnosis, therapy duration, associated corticotherapy and serum inflammatory parameters (ESR and CRP).

Institutional review board statement. The study was performed by respecting all ethical issues regarding the use of human tissues and the manipulation of paraffin embedded specimens, and ethical approval was obtained from the County Clinical Ethic Committee (Approval No. 2654/19.02.2019).

Skin biopsies primary processing and histopathologic diagnosis. Collected fresh skin biopsies were fixed in buffered formalin for 24$48 \mathrm{~h}$ followed by routine paraffin embedding protocol. Three $\mu \mathrm{m}$ thick sections were obtained from each block and mounted on glass slides. One slide from each case was stained with hematoxylin and eosin for histopathologic evaluation. Based on histopathology we selected slides for immunohistochemistry.

Immunohistochemistry was performed for the evaluation of CLIC1 expression on skin biopsies. All immunohistochemical procedures were performed by using the fully controlled automated immunohistochemistry workflow Bond Max Autostainer (Breckland, Linford Wood, UK). We used anti-CLIC1 (356.1) antibodies (Santa Cruz Biotechnology, SC 81873, Santa Cruz, CA, USA) with a dilution of 1:2000. One-hour incubation with CLIC1 primary antibody at room temperature was followed by applying the detection step using Bond Polymer Refine Detection Kit (Leica Microsystems). The final step of the immunohistochemical technique was visualization of the final product as a brown staining given by the use of 3,3 diaminobenzidine as chromogen and haematoxylin for nuclear counterstaining.

Microscopic evaluation and data analysis. Haematoxylin and eosinstained slides were evaluated for the identification of persistent histopathologic changes and to select slides for immunohistochemistry. Our study focused on the identification of CLIC1 expression in two compartments of the dermis: stromal compartment (especially on CLIC1 expression in stromal fibroblasts and inflammatory cells, CLIC1-S) and vascular compartment (CLIC1 expression on endothelial cells of dermal blood vessels, CLIC1-V). Based on CLIC1 expression on endothelial cells, we calculated the microvessel density, which was subsequently correlated with therapy type and other clinicopathologic parameters. All slides were scanned by using Desk Pannoramic Scanner (3D Histech, Budapest, Hungary) and they were stored in the Histology Department Digital Slides Library Case Center). Slide evaluation, picture capture, and processing were performed by using Pannoramic Viewer program (3D Histech). Evaluation of CLIC1 expression in stromal fibroblasts was performed by using the facilities of Cell Quant, at Quant Center (kindly provided 


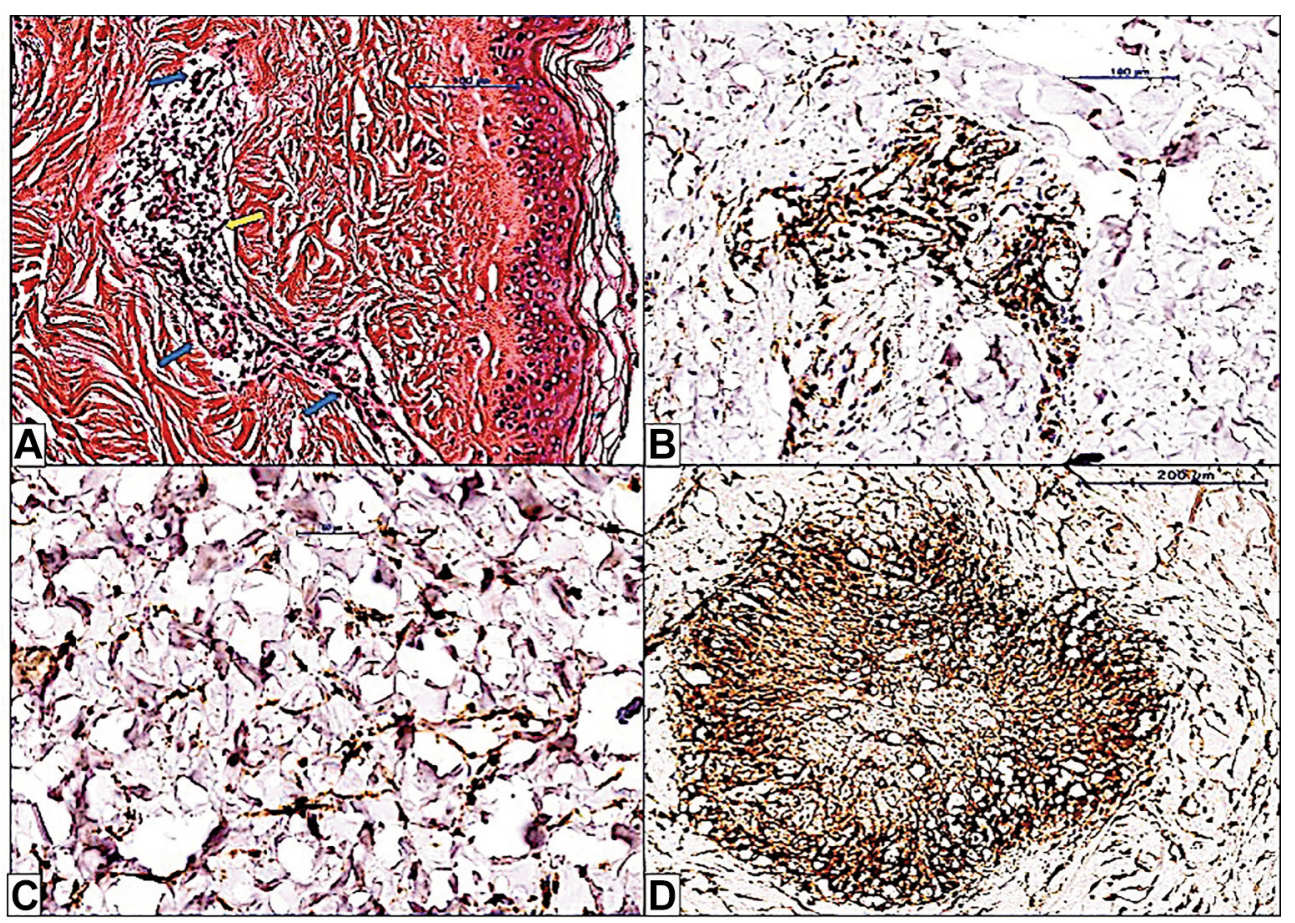

Figure 1. Skin biopsies from patients with rheumatoid arthritis (RA). (A) Microscopic view of vasculitis in skin biopsies of patients with RA. Note the branched capillaries network (blue arrows) and the massive inflammatory infiltrate (yellow arrow) surrounding it. (B) CLIC1 immunohistochemical expression in the endothelium of capillaries and inflammatory cells from vasculitis lesions. (C) CLIC1 positive stromal fibroblasts. (D) Rheumatoid nodule positive for CLIC1. Note the cytoplasmic and nuclear expression of CLIC1 in the outer part of the rheumatoid nodule, while the center is less stained.

by 3 DHistech). The evaluation score of this program is given by combination of intensity of CLIC1 expression and number of CLIC1 positive fibroblasts previously selected and defined by the pathologist. Also, CLIC1 expression in the endothelium of vessels was quantified by using the same semiautomated method of the Quant Center by counting CLIC1 positive blood vessels from skin biopsies.

Statistical analysis. Statistical analysis was performed by using JAMOVI software version 1.2.27.0. A $p$-value of $<0.05$ was considered statistically significant, while a $p$-value of $<0.001$ was considered strongly statistically significant. Charts and correlation tables included as part of the results section in this study were automatically generated by JAMOVI software.

\section{Results}

Histopathology and CLIC1 immunoexpression in RA and PsA skin biopsies. Vasculitis microscopic changes (Figure 1A) and classical rheumatoid nodules were observed on hematoxylin and eosin-stained skin biopsies. Microscopically, all selected cases were positive for CLIC1 by immunohistochemistry. CLIC1 was positive in the endothelium of skin blood vessels, stromal fibroblasts, and in the outer part of rheumatoid nodules. Inflammatory cells surrounding skin capillaries also expressed CLIC1 (Figure
1B-D). The inflammatory infiltrate was different from one case to another; it varied from scattered inflammatory cells with a heterogeneous distribution to a massive inflammatory infiltrate associated with groups of small skin capillaries lined by cells with the morphology of activated endothelial cells, which were positive for CLIC1. Based on this positivity in endothelial cells, we were able to quantify CLIC1 expression by performing counting of CLIC1 positive vessels, noted as CLIC1-V, and assessed their relationship to clinical, paraclinical, and therapeutic data. CLIC1 was expressed in blood vessel endothelium but not in the lymphatic endothelium of the skin. When we analysed CLIC1 expression in the vascular compartment of skin biopsies from RA and PsA patients, we observed that small blood vessels with CLIC1 positive endothelium had a heterogeneous morphology highly suggestive of an intense angiogenic process (we were able to detect microscopically angiogenic sprouts, intussusceptive angiogenesis, tube formation, and capillary like structures) also in the absence of inflammatory infiltrate. Most of the CLIC1 positive blood vessels had a branched morphology with a lumen lined by CLIC1 positive endothelial cells. The CLIC1 expression pattern was both nuclear and cytoplasmic (Figure 2). 


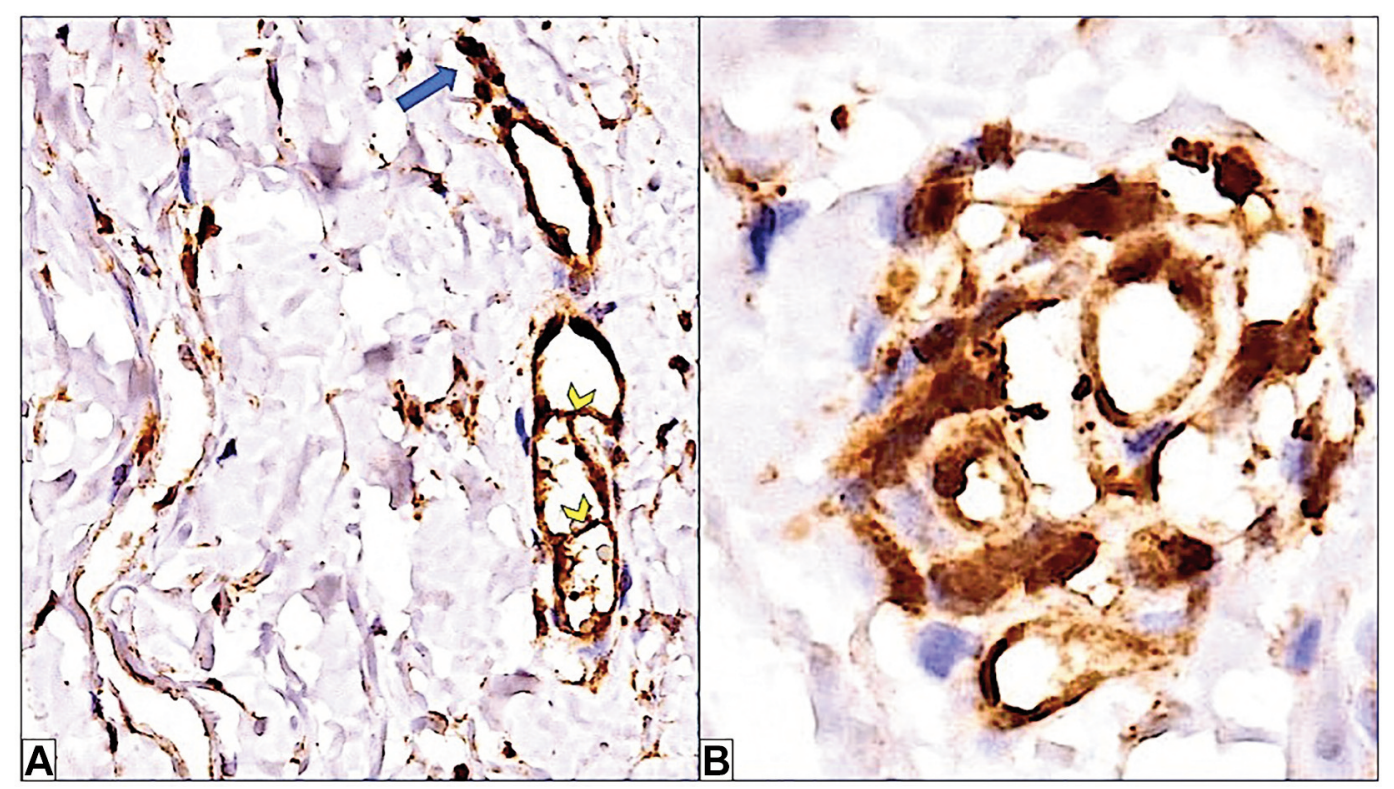

Figure 2. CLIC1 expression in the vascular compartment of the skin from patients with rheumatoid arthritis (RA). (A) CLIC1 positive vessels with morphology suggestive of activated endothelial cells with nuclear and cytoplasmic expression organized as sprouts emerging from pre-existing blood vessels (blue arrow). Vascular lumen split by CLIC1 positive intraluminal pillars (yellow arrowhead) highly suggestive for the presence of intussusceptive angiogenesis. (B) Groups of CLIC1 positive small capillaries arranged in a pseudo-glomerular pattern mimicking glomeruloid bodies usually found in highly angiogenic tissues but here in the skin biopsies from patients with RA. Note, also the nuclear and cytoplasmic expression of CLIC1 in endothelial cells.

Table I. Correlation matrix showing age-dependent CLIC1 immunoexpression in the stromal compartment (CLIC1-S) of the rheumatoid arthritis affected skin despite of the therapy received.

\begin{tabular}{llc}
\hline & & Age \\
\hline CLIC1-S & Pearson's r & $0.737 * *$ \\
& $p$-value & 0.001 \\
& $95 \%$ CI Upper & 0.903 \\
95\% CI Lower & 0.380 \\
& Spearman's rho & $0.932^{* * *}$ \\
& $p$-value & $<0.001$ \\
& Kendall's Tau B & $0.843^{* * *}$ \\
& $p$-value & $<0.001$ \\
\hline
\end{tabular}

CLIC1-S: Chloride intracellular channel protein 1 expression in stromal compartment. ${ }^{*} p<0.01, * * * p<0.001$, one-tailed.

Sprouting phenomenon with tip cells intensely positive for CLIC1 and intraluminal CLIC1 positive pillars were the main microscopic features indicating intense angiogenesis in skin biopsies from RA and PsA patients (Figure 2A and B).

Age and CLIC1 immunoexpression in skin biopsies from $R A$ and PsA patients. Age of the patients seems to differentially influence CLIC1-V and CLIC1-S immunoexpression in the skin of patients treated for RA. A strong correlation has been found
Table II. An inverse correlation was found between CLIC1 immunoexpression in stromal (CLIC1-S) and vascular (CLIC1-V) compartments.

\begin{tabular}{llc}
\hline & & CLIC1-V \\
\hline CLIC1-S & Pearson's r & $-0.544^{*}$ \\
& $p$-Value & 0.018 \\
& Spearman's rho & -0.135 \\
& p-Value & 0.315 \\
& Kendall's Tau B & -0.220 \\
& $p$-Value & 0.126 \\
\hline
\end{tabular}

CLIC1-S: Chloride intracellular channel protein 1 expression in stromal compartment; CLIC1-V: chloride intracellular channel protein 1 expression in vascular compartment. $* p<0.05$, one-tailed.

between age and high CLIC1-S immunoexpression $(p<0.001)$ for patients aged over 50 years compared with younger ones (Table I). Also, when we analyzed cases according to age, we observed that CLIC-S expression was partial and negatively correlated with CLIC-V immunoexpression $(p<0.018)$ in the dermal compartment of patients with RA (Table II). These findings suggest that older patients retain CLIC1 positive stromal cells in their skin but not CLIC1 positive blood vessels, independently of the therapy received. CLIC1-S persistence in 
Table III. Methotrexate (MTX) and leflunomide (LFN) therapy influence on CLIC1 expression in skin biopsies from rheumatoid arthritis patients (a) MTX therapy was significantly correlated with CLIC1-S but not with CLIC1-V. MTX therapy duration did not influence CLIC1 expression.

\begin{tabular}{|c|c|c|c|c|}
\hline & & CLIC1-S & CLIC1-V & MTX \\
\hline \multirow[t]{8}{*}{ CLIC1-V } & Pearson's r & -0.084 & - & \\
\hline & $p$-Value & 0.741 & - & \\
\hline & 95\% CI Upper & 0.399 & - & \\
\hline & 95\%CI Lower & -0.530 & - & \\
\hline & Spearman's rho & -0.387 & - & \\
\hline & $p$-Value & 0.113 & - & \\
\hline & Kendall's Tau B & -0.324 & - & \\
\hline & $p$-Value & 0.080 & - & \\
\hline \multirow[t]{8}{*}{ MTX } & Pearson's r & $0.556^{*}$ & 0.301 & - \\
\hline & $p$-Value & 0.016 & 0.225 & - \\
\hline & 95\% CI Upper & 0.812 & 0.673 & - \\
\hline & 95\%CI Lower & 0.121 & -0.193 & - \\
\hline & Spearman's rho & $0.652 * *$ & -0.131 & - \\
\hline & $p$-Value & 0.003 & 0.605 & - \\
\hline & Kendall's Tau B & $0.565 * *$ & -0.115 & - \\
\hline & $p$-Value & 0.007 & 0.589 & - \\
\hline \multirow[t]{8}{*}{ MTX-Months } & Pearson's r & -0.191 & 0.371 & 0.156 \\
\hline & $p$-Value & 0.449 & 0.130 & 0.536 \\
\hline & 95\%CI Upper & 0.303 & 0.714 & 0.581 \\
\hline & 95\%CI Lower & -0.604 & -0.116 & -0.335 \\
\hline & Spearman's rho & -0.365 & 0.419 & 0.137 \\
\hline & $p$-Value & 0.137 & 0.083 & 0.587 \\
\hline & Kendall's Tau B & -0.312 & 0.316 & 0.127 \\
\hline & $p$-Value & 0.111 & 0.110 & 0.571 \\
\hline
\end{tabular}

CLIC1-S: Chloride intracellular channel protein 1 expression in stromal compartment; CLIC1-V: chloride intracellular channel protein 1 expression in vascular compartment; MTX: methotrexate therapy; MTXMONTHS: therapy duration (months) with MTX. ${ }^{*} p<0.05, * * p<0.01$.

skin biopsies from older patients with RA may be considered as a poor prognostic factor. No significant correlation was detected between age, CLIC1-S $(p=0.777)$, and CLIC1-V $(p=0.702)$ for patients with PsA.

Effects of conventional therapies, corticotherapy, and biological therapies on CLIC1 expression in the skin stromal and vascular compartments from patients with RA and PsA. Methotrexate was used as first line therapy for treating patients with RA. Following MTX therapy, the increased expression of CLIC1 in the stromal compartment of the skin of RA patients was maintained (CLIC1-S, $p=0.016$ ), while CLIC1-V was not influenced by MTX therapy $(p=0.255)$ (Table III). Also, MTX therapy duration had no impact on both CLIC1-S $\quad(p=0.449)$ and CLIC1-V $\quad(p=0.130)$. Leflunomide (LFN) therapy in RA patients did not influence CLIC1-S but significantly reduced MVD as assessed by CLIC1-V immunoexpression $(p<0.001)$. Compared with MTX therapy whose duration did not influence CLIC1 expression, LFN therapy duration strongly decreased CLIC$\mathrm{V}$ immunoexpression $(p<0.001)$ (Table IV).
Table IV. Significant correlation between LFN dose and therapy duration and the decrease in CLIC1-V expression in skin biopsies from rheumatoid arthritis patients.

\begin{tabular}{|c|c|c|c|c|}
\hline & & CLIC1-S & CLIC1-V & LFN \\
\hline \multirow[t]{8}{*}{ CLIC1-V } & Pearson's r & -0.084 & - & \\
\hline & $p$-Value & 0.741 & - & \\
\hline & 95\% CI Upper & 0.399 & - & \\
\hline & 95\%CI Lower & -0.530 & - & \\
\hline & Spearman's rho & -0.387 & - & \\
\hline & $p$-Value & 0.113 & - & \\
\hline & Kendall's Tau B & -0.324 & - & \\
\hline & $p$-Value & 0.080 & - & \\
\hline \multirow[t]{8}{*}{ LFN } & Pearson's r & 0.082 & $-0.766 * * *$ & - \\
\hline & $p$-Value & 0.746 & $<0.001$ & - \\
\hline & 95\% CI Upper & 0.529 & -0.465 & - \\
\hline & 95\%CI Lower & -0.400 & -0.908 & - \\
\hline & Spearman's rho & 0.225 & $-0.770 * * *$ & - \\
\hline & $p$-Value & 0.370 & $<0.001$ & - \\
\hline & Kendall's Tau B & 0.159 & $-0.678 * * *$ & - \\
\hline & $p$-Value & 0.415 & $<0.001$ & - \\
\hline \multirow[t]{8}{*}{ LFN.Months } & Pearson's r & 0.148 & $-0.712 * * *$ & $0.635^{* *}$ \\
\hline & $p$-Value & 0.559 & $<0.001$ & 0.005 \\
\hline & 95\% CI Upper & 0.575 & -0.367 & 0.850 \\
\hline & 95\%CI Lower & -0.343 & -0.885 & 0.240 \\
\hline & Spearman's rho & $0.622 * *$ & $-0.810 * * *$ & $0.703 * *$ \\
\hline & $p$-Value & 0.006 & $<0.001$ & 0.001 \\
\hline & Kendall's Tau B & $0.605 * *$ & $-0.706^{* * *}$ & $0.611 * *$ \\
\hline & $p$-Value & 0.002 & $<0.001$ & 0.003 \\
\hline
\end{tabular}

CLIC1-S: Chloride intracellular channel protein 1 expression in stromal compartment; CLIC1-V: chloride intracellular channel protein 1 expression in vascular compartment; LFN: leflunomide therapy; LFNMONTHS: therapy duration (months) with LFN. ${ }^{* *} p<0.01$, ${ }^{* * *} p<0.001$.

Associated corticotherapy reduced CLIC1-S expression in a dose- and time-dependent manner $(p=0.006)$, but it did not influence dermal CLIC1-V. For skin biopsies derived from PsA patients, MTX therapy had a behaviour similar to RA; significantly correlated with CLIC1-S expression $(p=0.022)$ but had no influence on CLIC1-V expression. No other therapies influenced CLIC1-S and CLIC1-V immunoexpression in the skin from patients with PsA, which was sustained; no significant correlations were found. Biological therapies (humira and tofacitinib) had a dual impact on both CLIC1-S and CLIC1-V expression in skin biopsies from RA patients. Both therapies reduced not only CLIC1-S $(p<0.001)$ but also CLIC1-V ( $p=0.007)$ immunoexpression (Table V).

Serum inflammatory parameters such as erythrocyte sedimentation rate $(E S R)$ and $C$ reactive protein $(C R P)$ were usually normalized during the therapy applied. The correlation of serum inflammatory parameters, (usually used in RA as part of the pre- and post-therapy evaluation) with CLIC1-S and CLIC1-V was examined. We found that, despite of theESR and CRP normalisation an inverse 
Table V. Correlation matrix showing significant effects of biological therapies on CLIC1 immunoexpression in both vascular and stromal compartments of skin biopsies.

\begin{tabular}{|c|c|c|c|}
\hline & & CLIC1-V & CLIC1-S \\
\hline \multirow[t]{8}{*}{ CLIC1-V } & Pearson's r & - & \\
\hline & $p$-Value & - & \\
\hline & 95\% CI Upper & - & \\
\hline & 95\%CI Lower & - & \\
\hline & Spearman's rho & - & \\
\hline & $p$-Value & - & \\
\hline & Kendall's Tau B & - & \\
\hline & $p$-Value & - & \\
\hline \multirow[t]{8}{*}{ CLIC1-S } & Pearson's r & $0.984 * * *$ & - \\
\hline & $p$-Value & $<0.001$ & - \\
\hline & 95\%CI Upper & 0.998 & - \\
\hline & 95\%CI Lower & 0.859 & - \\
\hline & Spearman's rho & $1.000 * * *$ & - \\
\hline & $p$-Value & $<0.001$ & - \\
\hline & Kendall's Tau B & $1.000 *$ & - \\
\hline & $p$-Value & 0.012 & - \\
\hline \multirow[t]{8}{*}{ Biol therapy } & Pearson's r & $-0.982 * * *$ & $-0.933 * *$ \\
\hline & $p$-Value & $<0.001$ & 0.007 \\
\hline & 95\% CI Upper & -0.839 & -0.502 \\
\hline & 95\% CI Lower & -0.998 & -0.993 \\
\hline & Spearman's rho & $-0.866^{*}$ & $-0.866^{*}$ \\
\hline & $p$-Value & 0.026 & 0.026 \\
\hline & Kendall's Tau B & -0.816 & -0.816 \\
\hline & $p$-Value & 0.053 & 0.053 \\
\hline
\end{tabular}

CLIC1-S: Chloride intracellular channel protein 1 expression in stromal compartment; CLIC1-V: chloride intracellular channel protein 1 expression in vascular compartment; Biol Therapies: Biological Therapies (Humira, Tofacitinib). ${ }^{*} p<0.05, * * p<0.01, * * * p<0.001$.

correlation was detected between CLIC1-S and ESR $(p=0.0018)$ and CRP $(p=0.026)$ but not for CLIC1-V. This finding suggests that ESR and CRP normalization did not give a fully objective evaluation of the inflammation status in RA and the persistence of elevated CLIC1-S in patients' skin with RA may be considered (Table VI).

\section{Discussion}

RA is an autoimmune disease primarily affecting the joints but also other organs including the skin. Psoriasis, a well-known skin lesion, may be accompanied by PsA. Both diseases induce a general inflammatory response, developed through different mechanisms involving inflammasomes. Inflammasomes are stimulus-induced multimeric protein complexes of innate immunity (19), which have a high rate of polymorphism in RA and psoriasis $(20,21)$; they trigger an inflammatory response following their activation through heterogeneous complex mechanisms. NLRP3 rs35829419 or CARD8 rs2043211 polymorphisms support a proinflammatory state in about $8 \%$ of patients at the time of diagnosis, whereas the CARD 8
Table VI. ESR and CRP variability related to CLIC1-S and CLIC1-V in the skin of patients with rheumatoid arthritis. Note that CLIC1-S expression showed a significant negative correlation with both ESR and CRP.

\begin{tabular}{|c|c|c|c|c|}
\hline & & CLIC1-V & CLIC1-S & ESR \\
\hline \multirow[t]{8}{*}{ CLIC1-V } & Pearson's r & - & & \\
\hline & $p$-Value & - & & \\
\hline & 95\%CI Upper & - & & \\
\hline & 95\%CI Lower & - & & \\
\hline & Spearman's rho & - & & \\
\hline & $p$-Value & - & & \\
\hline & Kendall's Tau B & - & & \\
\hline & $p$-Value & - & & \\
\hline \multirow[t]{8}{*}{ CLIC1-S } & Pearson's r & -0.084 & - & \\
\hline & $p$-Value & 0.741 & - & \\
\hline & 95\% CI Upper & 0.399 & - & \\
\hline & 95\%CI Lower & -0.530 & - & \\
\hline & Spearman's rho & -0.387 & - & \\
\hline & $p$-Value & 0.113 & - & \\
\hline & Kendall's Tau B & -0.324 & - & \\
\hline & $p$-Value & 0.080 & - & \\
\hline \multirow[t]{8}{*}{ ESR } & Pearson's r & -0.137 & $-0.552 *$ & - \\
\hline & $p$-Value & 0.587 & 0.018 & - \\
\hline & 95\%CI Upper & 0.352 & -0.115 & - \\
\hline & 95\% CI Lower & -0.568 & -0.810 & - \\
\hline & Spearman's rho & -0.052 & -0.313 & - \\
\hline & $p$-Value & 0.839 & 0.206 & - \\
\hline & Kendall's Tau B & -0.031 & -0.182 & - \\
\hline & $p$-Value & 0.874 & 0.342 & - \\
\hline \multirow[t]{8}{*}{ CRP } & Pearson's r & -0.093 & $-0.523 *$ & $0.740 * * *$ \\
\hline & $p$-Value & 0.715 & 0.026 & $<0.001$ \\
\hline & 95\%CI Upper & 0.391 & -0.075 & 0.897 \\
\hline & 95\% CI Lower & -0.536 & -0.796 & 0.417 \\
\hline & Spearman's rho & 0.218 & $-0.478^{*}$ & $0.622 * *$ \\
\hline & $p$-Value & 0.384 & 0.045 & 0.006 \\
\hline & Kendall's Tau B & 0.191 & $-0.414 *$ & $0.562 *$ \\
\hline & $p$-Value & 0.368 & 0.049 & 0.010 \\
\hline
\end{tabular}

CLIC1-S: Chloride intracellular channel protein 1 expression in stromal compartment; CLIC1-V: chloride intracellular channel protein 1 expression in vascular compartment; ESR: erythrocytes sedimentation rate; CRP: C reactive protein. ${ }^{*} p<0.05, * * p<0.01, * * * p<0.001$.

rs2043211 TT genotype seems to be strongly involved in resistance to therapy by continuing to promote a proinflammatory state even 6 months after the beginning of therapy (20). CLIC1 seems to be one of the most important factors involved in NRLP3 inflammasome activation reported to be highly responsible for the inflammatory events in RA (12, 22). The involvement of CLIC1 in arthritis was indirectly shown by Jiang et al. (2), who created $\mathrm{CLIC1}^{-/-}$mice having macrophages with an elevated phagosome $\mathrm{pH}$. Because of this peculiarity, such mice were not able to develop macrophagedependent immune-complex mediated arthritis (2). Here, we report for the first time the presence and expression of CLIC1 in human rheumatoid nodules. This microscopic evidence 
supports CLIC1 involvement in the pathogenesis of RA in humans most probably through NRLP3 activation. This is in accordance with the previous findings of Heruth et al., who demonstrated that CLIC1 is exclusively expressed in human rheumatoid arthritis fibroblasts but not in human normal synovial fibroblasts (23). Based on this observation and together with the inflammatory events found in the RA patients' skin, we may hypothesize that CLIC1 positive skin fibroblasts (noted in our study with CLIC1-S) may be considered as an indicator of active disease and may be used to assess the impact of different therapies received by our patients during disease progression on CLIC1 expression.

The inflammation milieu induces endothelial cell activation and triggers angiogenic processes. CLIC1 translocation in endothelial cells has been observed in malignant tumors such as glioblastoma (24), suggesting that it may be involved in angiogenesis, tumor progression, and metastasis $(25,26)$. Immunohistochemical expression of CLIC1 in the tumor blood vessel endothelium has been recently reported by Raica et al. (27). Our team assessed MVD by using for the first time CLIC1 endothelial immunoexpression as a marker of activated endothelium in newly formed breast cancer blood vessels. We applied the same model for assessing CLIC1 positive blood vessels (noted here with CLIC1-V) in skin biopsies from RA and PsA patients, considering that endothelial cells are highly activated by inflammation if they express CLIC1.

It is well known that RA and PsA patients have no favorable response to the same drugs during disease progression. Thus, for most of them, there is a need to implement second line therapy associated or not with corticotherapy, and the use of antibody-based targeted therapies. Most of the RA and PsA patients are treated with methotrexate (MTX) as first line therapy, as in our study. Our results showed that MTX had no influence on CLIC1-S expression in skin biopsies from RA and PsA patients. This finding suggested that CLIC1 may be a potential factor of development of chemoresistance to MTX, which may explain in part why patients become unresponsive to MTX during RA and PsA disease progression. Recent data confirmed CLIC1 involvement in the development of MTX chemoresistance by tumor cells (28) and highlighted also the molecular mechanism of CLIC1-induced chemoresistance (29).

Leflunomide therapy negatively influenced CLIC1-V expression in our study. Indirect evidence, which may explain our results, is found in the literature but none of them are related to RA or PsA. Leflunomide is a NF-kB and TNF- $\alpha$ inhibitor, two factors involved in inflammationinduced angiogenesis in RA and PsA. NF- $\mathrm{kB}$ is positively regulated by CLIC1 (30), which also induces TNF- $\alpha$, expression in endothelial cells promoting vascular inflammation (18). In vitro, the strongest activator of $\mathrm{NF}-\mathrm{kB}$ in endothelial cells appears to be TNF $\alpha$ (31).
CLIC1-V was significantly decreased in leflunomide-treated patients, most probably due to inhibition of NF-kB and TNF$\alpha$, which are usually found in high levels in these diseases.

Biological therapies used in our study act on both the stromal and vascular compartment in skin biopsies from RA patients. Adalimumab (humira) exerts anti TNF- $\alpha$ effects, whereas tofacitinib acts on JAK/STAT3 pathway. Both drugs reduced CLIC1-S and CLIC1-V expression in skin biopsies from RA patients but no evidence of interaction of CLIC1 with JAK/STAT3 has been reported. The decrease in the expression of CLIC1-V and CLIC1-S following tofacitinib treatment may be explained by an indirect mechanism of lymphocyte inhibition and a subsequent change in the inflammatory microenvironment.

The presence of CLIC1 in the endothelium of blood vessels where we were able to observe sprouting and/or intussusceptive angiogenesis microscopic patterns suggested that CLIC1 may promote the angiogenesis. There has been indirect evidence of CLIC1 involvement in endothelial cell activation from blood vessels found in tumor and non-tumor conditions (32-34). In vitro studies performed by Tung and Kitajewski have shown that CLIC1 is involved in most steps of the angiogenic process by promoting endothelial cell migration, proliferation, and their ability to form capillarylike structures and branches (32). These data have not yet been validated on human tissues. Our observations regarding the presence of blood vessels having CLIC1 positive sproutlike structures and also CLIC1 positive intraluminal pillars are in concordance with previous in vitro findings by Tung and Kitajewski and are the first microscopic evidence of CLIC1 involvement in inflammation-induced angiogenesis in skin biopsies of patients with RA and PsA. Although there is only indirect evidence on the involvement of CLIC1 in angiogenesis, TNF- $\alpha$ and JAK/STAT3 pathway (the main targets for biological therapies in RA and PsA) are known to be key players in the angiogenic process $(35,36)$ by endothelial cell activation. For patients with RA enrolled in our study and treated with biological therapies (adalimumab as anti-TNF- $\alpha$ agent and tofacitinib as anti-JAK/STAT3 pathway) we found a significant decrease in CLIC1 immunoexpression in the vascular compartment followed by a decrease in microvessel density. Our data support a strong interrelation between CLIC1, TNF- $\alpha$, and the JAK/STAT3 pathway in RA, however, further studies are needed to fully elucidate the mechanism of such interrelation.

\section{Conclusion}

The present study is the first report on CLIC1 expression in rheumatoid nodules, and stromal and vascular compartments in skin biopsies from patients with RA and PsA. Both diseases are characterised by inflammation involving several organs, including the skin, and it seems that CLIC1 is deeply involved 
in the inflammatory skin lesions of patients with RA and PsA. We report here that RA and PsA therapeutic agents may differentially influence CLIC1 skin expression, and its dynamic changes are independent of serum inflammatory markers. Thus, we may conclude that CLIC1 assessment in skin biopsies from patients with RA and PsA may be used as a potential tool for predicting the general inflammatory state and therapy response. Currently, no data regarding CLIC1 serum evaluation are available for RA and PsA despite of its function as one of the potent factors in promoting inflammation. Our data strongly support extensive evaluation of CLIC1 in RA as a potential new tissue and serum marker of inflammation assessment and predictive tool for therapy response.

\section{Funding}

The APC was funded by Victor Babes University of Medicine and Pharmacy Timisoara Romania, from special funds allocated for research and Doctoral School Ph.D. students.

\section{Conflicts of Interest}

The Authors declare no conflicts of interest. The funders had no role in the design of the study; the collection, analyses, or interpretation of data; in the writing of the manuscript, or in the decision to publish the results.

\section{Authors' Contributions}

Conceptualization, L.B. and M.C.; methodology, O.S.C.; validation, A.M.C., investigation, L.B. and A.M.C.; writing-original draft preparation, L.B. and M.C.; writing-review and editing, A.M.C.; supervision, M.R., CA- investigation. All Authors have read and agreed to the published version of the manuscript.

\section{Acknowledgements}

The Authors are grateful to Ciprian Onica and Patricia Berzava for their excellent technical support.

\section{References}

1 Gururaja Rao S, Ponnalagu D, Patel NJ and Singh H: Three decades of chloride intracellular channel proteins: from organelle to organ physiology. Curr Protoc Pharmacol 80(1): 11.21.111.21.17, 2018. PMID: 30040212. DOI: $10.1002 / \mathrm{cpph} .36$

2 Jiang L, Salao K, Li H, Rybicka JM, Yates RM, Luo XW, Shi XX, Kuffner T, Tsai VW, Husaini Y, Wu L, Brown DA, Grewal $\mathrm{T}$, Brown LJ, Curmi PM and Breit SN: Intracellular chloride channel protein CLIC1 regulates macrophage function through modulation of phagosomal acidification. J Cell Sci 125(Pt 22): 5479-5488, 2012. PMID: 22956539. DOI: 10.1242/jcs.110072

3 Valenzuela SM, Mazzanti M, Tonini R, Qiu MR, Warton K, Musgrove EA, Campbell TJ and Breit SN: The nuclear chloride ion channel NCC27 is involved in regulation of the cell cycle. $\mathrm{J}$ Physiol 529 Pt 3: 541-552, 2000. PMID: 11195932. DOI: 10.1111/j.1469-7793.2000.00541.x
4 The human protein atlas. Available at: https://www.proteinatlas. org/ENSG00000213719-CLIC1/celltype [Last accessed on May 25, 2021]

5 Carlini V, Verduci I, Cianci F, Cannavale G, Fenoglio C, Galimberti D and Mazzanti M: CLIC1 protein accumulates in circulating monocyte membrane during neurodegeneration. Int J Mol Sci 21(4): 1484, 2020. PMID: 32098256. DOI: 10.3390/ ijms 21041484

6 Zhu J, Xu Y, Ren G, Hu X, Wang C, Yang Z, Li Z, Mao W and Lu D: Tanshinone IIA Sodium sulfonate regulates antioxidant system, inflammation, and endothelial dysfunction in atherosclerosis by downregulation of CLIC1. Eur J Pharmacol 815: 427-436, 2017. PMID: 28970012. DOI: 10.1016/j.ejphar. 2017.09 .047

7 Huang ZX, Tan JH, Li TW, Deng WM, Qiu KW, Liao ZT and Zeng ZQ: Influence of sinomenine on protein profiles of peripheral blood mononuclear cells from ankylosing spondylitis patients: a pharmacoproteomics study. Chin Med J (Engl) 126(19): 3645-3650, 2013. PMID: 24112157.

8 Adelmann TG, Camerota TC, Ceausu AR, Cimpean AM, Mazzanti M and Raica M: Chloride intracellular channel protein 1 (CLIC1) ts over-expressed in muscle invasive urinary bladder cancer. Anticancer Res 40(12): 6879-6884, 2020. PMID: 33288580. DOI: 10.21873 /anticanres.14710

9 Nesiu A, Cimpean AM, Ceausu RA, Adile A, Ioiart I, Porta C, Mazzanti M, Camerota TC and Raica M: Intracellular chloride ion channel protein-1 expression in clear cell renal cell carcinoma. Cancer Genomics Proteomics 16(4): 299-307, 2019. PMID: 31243111. DOI: 10.21873/cgp.20135

10 Peng JM, Lin SH, Yu MC and Hsieh SY: CLIC1 recruits PIP5K1A/C to induce cell-matrix adhesions for tumor metastasis. J Clin Invest 131(1): e133525, 2021. PMID: 33079727. DOI: 10.1172/JCI133525

11 Gururaja Rao S, Patel NJ and Singh H: Intracellular chloride channels: Novel biomarkers in diseases. Front Physiol 11: 96, 2020. PMID: 32116799. DOI: 10.3389/fphys.2020.00096

12 Domingo-Fernández R, Coll RC, Kearney J, Breit S and O'Neill LAJ: The intracellular chloride channel proteins CLIC1 and CLIC4 induce IL-1 $\beta$ transcription and activate the NLRP3 inflammasome. J Biol Chem 292(29): 12077-12087, 2017. PMID: 28576828. DOI: 10.1074/jbc.M117.797126

13 Du H, Wang Y, Zeng Y, Huang X, Liu D, Ye L, Li Y, Chen X, Liu T, Li H, Wu J, Yu Q, Wu Y and Jie L: Tanshinone IIA suppresses proliferation and inflammatory cytokine production of synovial fibroblasts from rheumatoid arthritis patients induced by TNF- $\alpha$ and attenuates the inflammatory response in AIA mice. Front Pharmacol 11: 568, 2020. PMID: 32499694. DOI: 10.3389/fphar.2020.00568

14 Berghea F, Vlad V, Palanciuc L, Micu M, Bojinca V, Vreju F, Enache L, Copotoiu M, Kosevoi A, Serban T, Stanciu D, Abobului M, Borangiu A, Berghea CE, Balanescu AR and Ionescu R: Quantitative Doppler in musculoskeletal ultrasonography - suboptimal performance of both experienced and in-training sonographers in selection of the highest Doppler signal image from cine-loops. Med Ultrason 19(3): 282-287, 2017. PMID: 28845494. DOI: 10.11152/mu933

15 Sharma A and Albert D: Dermatologic manifestations of rheumatoid arthritis. Rheumatology (Sunnyvale) 5(3): 168, 2015. DOI: $10.4172 / 2161-1149.1000168$ 
16 Sánchez-Cárdenas G, Contreras-Yáñez I, Guaracha-Basáñez G, Pacheco-Santiago LD, Méndez-Flores S, Barrera-Vargas A, Merayo-Chalico J, Domínguez-Cherit J and Pascual-Ramos V: Cutaneous manifestations are frequent and diverse among patients with rheumatoid arthritis and impact their quality of life: a cross-sectional study in a cohort of patients with recent-onset disease. Clin Rheumatol, 2021. PMID: 33651260. DOI: 10.1007/s10067-021-05664-0

17 Xue Y, Cohen JM, Wright NA and Merola JF: Skin signs of rheumatoid arthritis and its therapy-induced cutaneous side effects. Am J Clin Dermatol 17(2): 147-162, 2016. PMID: 26649439. DOI: 10.1007/s40257-015-0167-Z

$18 \mathrm{Xu} \mathrm{Y,} \mathrm{Zhu} \mathrm{J,} \mathrm{Hu} \mathrm{X,} \mathrm{Wang} \mathrm{C,} \mathrm{Lu} \mathrm{D,} \mathrm{Gong} \mathrm{C,} \mathrm{Yang} \mathrm{J} \mathrm{and} \mathrm{Zong}$ L: CLIC1 Inhibition attenuates vascular inflammation, oxidative stress, and endothelial injury. PLoS One 11(11): e0166790, 2016. PMID: 27861612. DOI: 10.1371/journal.pone.0166790

19 Fusco R, Siracusa R, Genovese T, Cuzzocrea S and Di Paola R: Focus on the role of NLRP3 inflammasome in diseases. Int J Mol Sci 21(12): 4223, 2020. PMID: 32545788. DOI: 10.3390/ ijms21124223

20 Jenko B, Praprotnik S, Tomšic M and Dolžan V: NLRP3 and CARD 8 polymorphisms influence higher disease activity in rheumatoid arthritis. J Med Biochem 35(3): 319-323, 2016. PMID: 28356883. DOI: 10.1515/jomb-2016-0008

21 Fenini G, Karakaya T, Hennig P, Di Filippo M and Beer HD: The NLRP1 inflammasome in human skin and beyond. Int J Mol Sci 21(13): 4788, 2020. PMID: 32640751. DOI: 10.3390/ ijms 21134788

22 Tang T, Lang X, Xu C, Wang X, Gong T, Yang Y, Cui J, Bai L, Wang J, Jiang W and Zhou R: CLICs-dependent chloride efflux is an essential and proximal upstream event for NLRP3 inflammasome activation. Nat Commun 8(1): 202, 2017. PMID: 28779175. DOI: 10.1038/s41467-017-00227-X

23 Heruth DP, Gibson M, Grigoryev DN, Zhang LQ and Ye SQ: RNA-seq analysis of synovial fibroblasts brings new insights into rheumatoid arthritis. Cell Biosci 2(1): 43, 2012. PMID: 23259760. DOI: $10.1186 / 2045-3701-2-43$

24 Thuringer D, Chanteloup G, Winckler P and Garrido C: The vesicular transfer of CLIC1 from glioblastoma to microvascular endothelial cells requires TRPM7. Oncotarget 9(70): 3330233311, 2018. PMID: 30279961. DOI: 10.18632/oncotarget. 26048

25 Geng HY, Feng ZJ, Zhang JJ and Li GY: Exosomal CLIC1 released by CLL promotes HUVECs angiogenesis by regulating ITGß1-MAPK/ERK axis. Kaohsiung J Med Sci 37(3): 226-235, 2021. PMID: 32841520. DOI: 10.1002/kjm2.12287

26 Gurski LA, Knowles LM, Basse PH, Maranchie JK, Watkins SC and Pilch J: Relocation of CLIC1 promotes tumor cell invasion and colonization of fibrin. Mol Cancer Res 13(2): 273-280, 2015. PMID: 25205595. DOI: $10.1158 / 1541-7786 . M C R-14-$ 0249

27 Raica M, Ceausu AR, Cimpean AM, ComŞa Ş and Sarb S: Chloride intracellular channel protein 1 (CLIC1), E-cadherin and P-cadherin define distinct subclasses of HER2, luminal B and triple-negative breast cancer. Anticancer Res 41(2): 795-802, 2021. PMID: 33517284. DOI: 10.21873/anticanres.14831
$28 \mathrm{Wu} \mathrm{J}$ and Wang D: CLIC1 induces drug resistance in human choriocarcinoma through positive regulation of MRP1. Oncol Res 25(6): 863-871, 2017. PMID: 27983917. DOI: 10.3727/ 096504016X14772315906527

29 Kischel P, Girault A, Rodat-Despoix L, Chamlali M, Radoslavova S, Abou Daya H, Lefebvre T, Foulon A, Rybarczyk P, Hague F, Dhennin-Duthille I, Gautier M and OuadidAhidouch H: Ion channels: New actors playing in chemotherapeutic resistance. Cancers (Basel) 11(3): 376, 2019. PMID: 30884858. DOI: 10.3390/cancers11030376

30 Wang W, Li X, Xu Y, Guo W, Yu H, Zhang L, Wang Y and Chen $\mathrm{X}$ : Acetylation-stabilized chloride intracellular channel 1 exerts a tumor-promoting effect on cervical cancer cells by activating NF-kB. Cell Oncol (Dordr), 2021. PMID: 33469837. DOI: 10.1007/s13402-020-00582-w

31 Mussbacher M, Salzmann M, Brostjan C, Hoesel B, Schoergenhofer C, Datler H, Hohensinner P, Basílio J, Petzelbauer P, Assinger A and Schmid JA: Cell type-specific roles of NF-kB linking inflammation and thrombosis. Front Immunol 10: 85, 2019. PMID: 30778349. DOI: 10.3389/ fimmu.2019.00085

32 Tung JJ and Kitajewski J: Chloride intracellular channel 1 functions in endothelial cell growth and migration. J Angiogenes Res 2: 23, 2010. PMID: 21040583. DOI: 10.1186/2040-2384-2-23

33 Knowles LM, Malik G, Hood BL, Conrads TP and Pilch J: CLT1 targets angiogenic endothelium through CLIC1 and fibronectin. Angiogenesis 15(1): 115-129, 2012. PMID: 22203240. DOI: $10.1007 / \mathrm{s} 10456-011-9247-8$

34 Biasiotta A, D'Arcangelo D, Passarelli F, Nicodemi EM and Facchiano A: Ion channels expression and function are strongly modified in solid tumors and vascular malformations. J Transl Med 14(1): 285, 2016. PMID: 27716384. DOI: 10.1186/s12967016-1038-y

35 Tanaka K, Watanabe M, Tanigaki S, Iwashita M and Kobayashi Y: Tumor necrosis factor- $\alpha$ regulates angiogenesis of BeWo cells via synergy of PlGF/VEGFR1 and VEGF-A/VEGFR2 axes. Placenta 74: 20-27, 2018. PMID: 30591201. DOI: 10.1016/ j.placenta.2018.12.009

36 Zegeye MM, Lindkvist M, Fälker K, Kumawat AK, Paramel G, Grenegård M, Sirsjö A and Ljungberg LU: Activation of the JAK/STAT3 and PI3K/AKT pathways are crucial for IL-6 transsignaling-mediated pro-inflammatory response in human vascular endothelial cells. Cell Commun Signal 16(1): 55, 2018. PMID: 30185178. DOI: 10.1186/s12964-018-0268-4
Received May 8, 2021

Revised May 26, 2021

Accepted May 31, 2021 